EPJ Web of Conferences 16, 07004 (2011)

DOI: $10.1051 /$ epjconf/20111607004

(C) Owned by the authors, published by EDP Sciences, 2011

\title{
The new worlds observer: The astrophysics strategic mission concept study
}

\author{
W. Cash ${ }^{1}$ and the New Worlds Team ${ }^{2}$ \\ ${ }^{1}$ University of Colorado \\ ${ }^{2}$ See Table 1
}

\begin{abstract}
We present some results of the Astrophysics Strategic Mission Concept Study for the New Worlds Observer (NWO). We show that the use of starshades is the most effective and affordable path to mapping and understanding our neighboring planetary systems, to opening the search for life outside our solar system, while serving the needs of the greater astronomy community. A starshade-based mission can be implemented immediately with a near term program of technology demonstration.
\end{abstract}

\section{THE MISSION}

The New Worlds Observer (NWO) is a mission concept for direct observation and study of exoplanets all the way down to Earth-like objects. Its goal is very simple: map the planetary systems of the nearby stars, search for habitable planets, and look for signs of life. An Astrophysics Strategic Mission Concept Study (ASMCS) has just been completed, and this paper summarizes some of the results of that study [1]. The many people of the New Worlds study team who contributed to this study and thus to this paper are listed in Table 1, along with an indicator of their roles.

Is Earth a unique outpost of life in a vast and empty Universe? How did planets come into being and why are they in their current state? What are the circumstances under which life arises, and how common is it? NASA can definitively address these questions in the coming decade with the New Worlds Observer (NWO).

Hundreds of giant exoplanets have now been detected and improvements in technology are moving the detection limits to smaller and smaller masses. NWO can discover Earth-like planets, but detecting their existence is just the beginning: only spectroscopy of planets in the habitable zones of dozens of stars can answer the question of how common life is in the Universe. A facility capable of finding and characterizing terrestrial planets requires that the starlight be suppressed by a factor of at least $10^{10}$ to enable the planet's light to be seen against the light of its host sun. This suppression needs to be confined within tens of milliarcseconds (mas) so that the planet's light is not blocked. Direct imaging with NWO will reveal most of the planets in an extrasolar system in just a single exposure. Through spectroscopy, we can determine the nature of each planet discovered.

The NWO mission concept (Fig. 1) can do all of this and more. Full suppression of the starlight before it enters the aperture relieves the telescope of demanding requirements such as ultra-high quality wave front correction and stray light control. The NWO telescope requires only diffraction-limited wavefront quality. This design results in a clean separation of light suppression and light collection. The starshade is a passive mechanical structure that only has centimeter-level requirements on the edge, but not over the surface. Integrated development of NWO could start today.

The NWO mission is illustrated in Figure 2. Two launch vehicles take the $50 \mathrm{~m}$ starshade and the $4 \mathrm{~m}$ telescope to L2, where they enter a halo orbit. The two spacecraft are separated by $\sim 80,000 \mathrm{~km}$.

This is an Open Access article distributed under the terms of the Creative Commons Attribution-Noncommercial License 3.0, which permits unrestricted use, distribution, and reproduction in any noncommercial medium, provided the original work is properly cited. 
EPJ Web of Conferences

Table 1. The New Worlds Observer study team.

\begin{tabular}{|c|c|c|}
\hline Co-author & Institution & Study Role \\
\hline Steve Kendrick & Ball & Optics and Telescope Design \\
\hline Charley Noecker & Ball & Mission Design Telescope Lead \\
\hline John Bally & $\mathrm{CU}$ & Science Team \\
\hline Julia DeMarines & $\mathrm{CU}$ & Outreach Education \\
\hline James Green & $\mathrm{CU}$ & Science, Instrument Design \\
\hline Phil Oakley & $\mathrm{CU}$ & Science Team \\
\hline Ann Shipley & $\mathrm{CU}$ & Starshade Laboratory Testing \\
\hline Scott Benson & GRC & NEXT Propulsion System Lead \\
\hline Steve Oleson & GRC & NEXT Propulsion System \\
\hline David Content & GSFC & Laboratory Testing \\
\hline Dave Folta & GSFC & Flight Dynamics \\
\hline Sharon Garrison & GSFC & Study Management \\
\hline Keith Gendreau & GSFC & Science System Engineer \\
\hline Kate Hartman & GSFC & Study Manager \\
\hline Joe Howard & GSFC & Optical Design \\
\hline Tupper Hyde & GSFC & Technology Lead \\
\hline Darryl Lakins & GSFC & Lead System Engineer \\
\hline Jesse Leitner & GSFC & Formation Flying Systems \\
\hline Doug Leviton & GSFC & Optics, Instrument, Lab Testing \\
\hline Rich Luquette & GSFC & Formation Flying Systems \\
\hline Bill Oegerley & GSFC & Science Team \\
\hline Karen Richon & GSFC & Orbits, Navigation lead \\
\hline Aki Roberge & GSFC & Science Team \\
\hline Steve Tompkins & GSFC & Ground Systems Mission Ops \\
\hline June Tveekrem & GSFC & Optics, Stray Light \\
\hline Bruce Woodgate & GSFC & Instruments \\
\hline Maggie Turnbull & GSI & Science Team Lead \\
\hline Dean Dailey & NGC & Mechanism and Structures Lead \\
\hline Kent Decker & NGC & Power Subsystem Lead \\
\hline Reza Dehmohseni & NGC & ACS/GN\&C Lead \\
\hline Brian Gaugh & NGC & Thermal Control Subsystem Lead \\
\hline Tiffany Glassman & NGC & Science team/Starshade Analysis \\
\hline Mickey Haney & NGC & Comm System Lead \\
\hline Reem Hejal & NGC & Dynamics Lead \\
\hline Chuck Lillie & NGC & Senior Advisor \\
\hline Amy Lo & NGC & Deput PI, Mission Design \\
\hline David O'Conner & NGC & Starshade ACS Lead \\
\hline Gina Oleas & NGC & System Engineer \\
\hline Ron Polidan & NGC & Senior Advisor \\
\hline Rocco Samuele & NGC & Starshade Laboratory Testing \\
\hline Stephen Shields & NGC & Mechanical Design Integration \\
\hline James Shirvanian & NGC & Formation Flying Conops \\
\hline David SooHoo & NGC & Propulsion \\
\hline Giovanna Tinetti & UCL & Science Team \\
\hline Bryan Dorland & USNO & JMAP Instrument PI \\
\hline Rachel Dudik & USNO & JMAPS Instrument \\
\hline Ralph Gaume & USNO & Astrometry \\
\hline Brian Mason & USNO & Science Team, astrometry \\
\hline
\end{tabular}




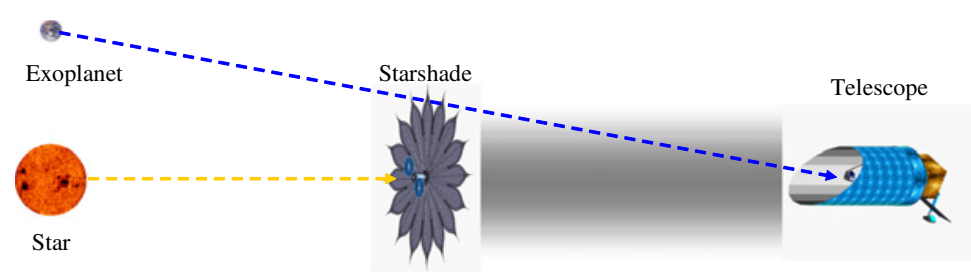

Figure 1. NWO's cost-effective starshade shadows the telescope from the star, while light from a terrestrial exoplanet passes the edge of the starshade unimpeded.

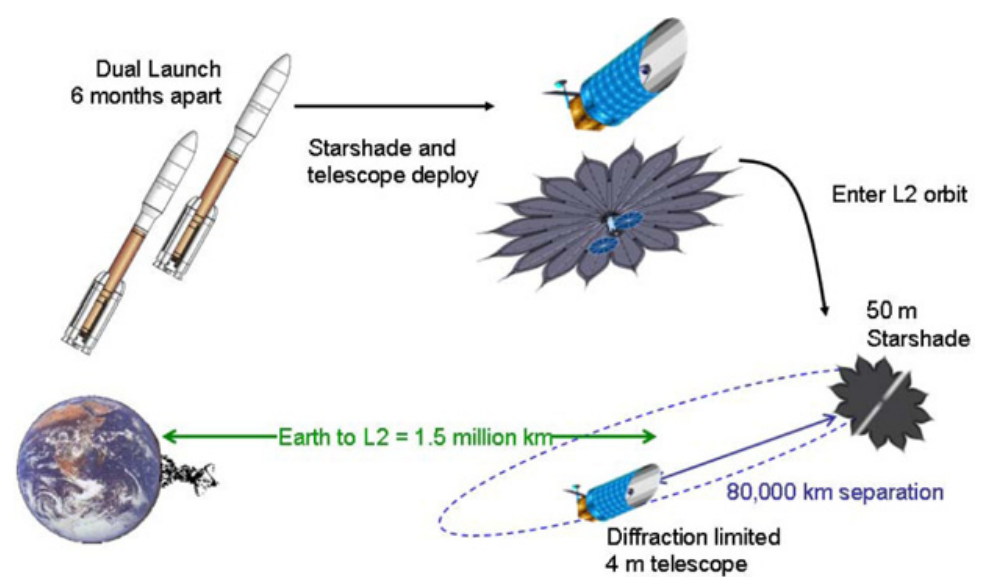

Figure 2. Employing existing technology, NWO uses a $4 \mathrm{~m}$ telescope and a $50 \mathrm{~m}$ starshade orbiting around the Sun-Earth L2 point to image and characterize terrestrial planets.

The starshade moves relative to the telescope to occult target stars. The average exoplanet observing cycle is $\sim 2$ weeks per star, with the capability of more than 100 cycles over a 5 year mission.

The study has shown that starshades are an extendable technology. Larger starshades can accommodate successively larger telescopes, freeing the telescope to adopt architectures such as segmented mirrors and central obstructions that are not available to other direct-imaging techniques. We took a detailed look at the technology necessary to make the New Worlds Observer a reality: most of this technology already exists. What is new for NWO is that these existing technologies have not been combined in this particular way before. Technology development for NWO can be rapidly implemented to lead to a flight program within a few years, with well-understood and controlled risks. The launch of a starshade can be envisioned to happen in as little as six years. The cost of a starshade-only mission is in the medium cost category. The cost of a $4 \mathrm{~m}$ UVOIR observatory is, unsurprisingly, in the flagship range.

The idea of a starshade is not new [2], but eliminating light diffracting around an external occulter for imaging Earth-like planets has been impractical [3, 4]. Recently, Cash [5] found an apodization function that made such a system practical with today's technology. Shown in Figure 7, the starshade is an opaque screen that sits in the line of sight from the telescope to the star. If the starshade is sufficiently distant, it will subtend a small angle, blotting out the star's light while allowing the exoplanet light to pass unobscured past the edge. A detailed requirements document for the Starshade was generated. Our science goals are taken from the TPF STDT report, and the NWO mission is designed to fulfill and exceed those requirements.

Cash's offset hyper-Gaussian apodization function reduces diffraction by many orders of magnitude; Figure 3 shows the parameters of this apodization function. A starshade with $2(a+b)=50 \mathrm{~m}$ (the 


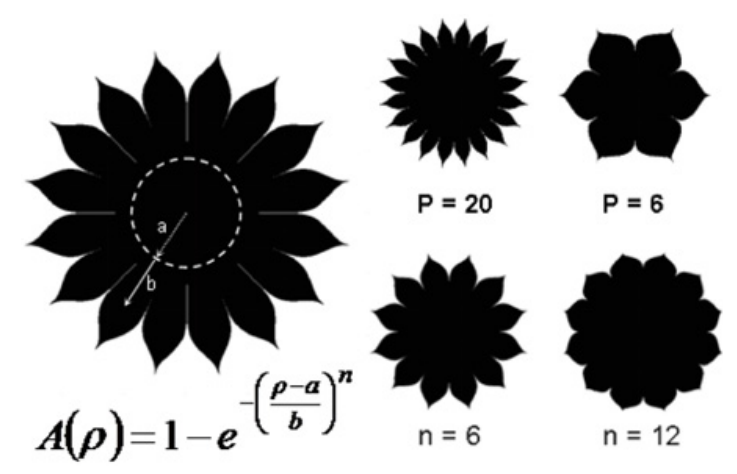

Figure 3. The apodization function, $\mathrm{A}(\rho)$, describes the shape of the starshade and can be optimized for suppression level, wavelength range, shadow size, and IWA.

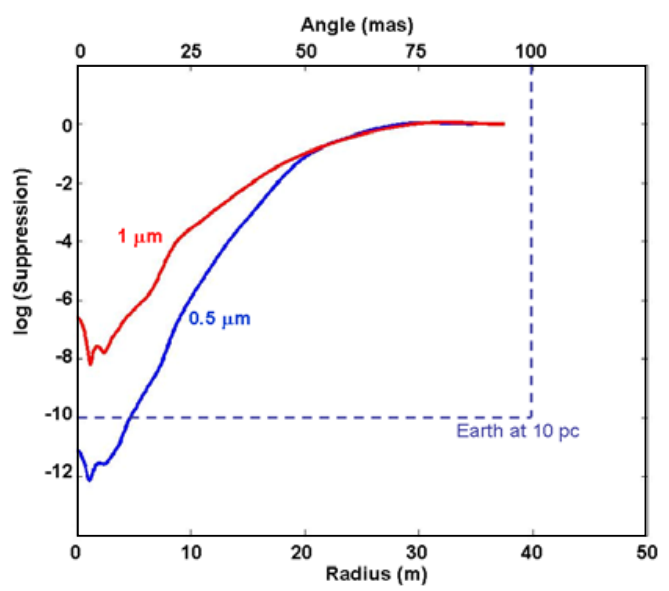

Figure 4. Light suppression falls by $>$ ten orders of magnitude across a shadow radius of just $20 \mathrm{~m}$, allowing observations of planets as close as 50 mas.

effective diameter), operating $\sim 80,000 \mathrm{~km}$ from a $4 \mathrm{~m}$ telescope is capable of $10^{10}$ starlight suppression within 50 mas for wavelengths from 0.1 to $1 \mu \mathrm{m}$. Our studies of the starshade in the past four years have shown that the optimal petal number for NWO is $\mathrm{P}=16$, a balance between starshade mass and shadow diameter. The hypergaussian parameter is optimized at $n=6$ over the wavelength range. Four independent software codes have been developed to simulate starshade performance. Figure 4 shows the suppression efficiency of the baseline starshade design as a function of both shadow radius and angular offset for two representative wavelengths.

We have chosen to baseline a $4 \mathrm{~m}$ telescope for its ability to resolve the exoplanet from the background, and because it is the breakpoint for a monolithic mirror fitting inside existing launch vehicles and using existing facilities. While it is larger than HST, this telescope has roughly the same tolerances. The optical design of the telescope is straightforward. This is primarily because the starlight from the target stars will be fully suppressed, so there are no special requirements on the optical train as there are for internal coronagraphic techniques. For example, segmented mirrors and any mirror coating may be used. The primary mirror configuration is still being studied (monolith vs. segmented) but our current baseline is a monolith. A $4 \mathrm{~m} \mathrm{UV/Optical/near-IR} \mathrm{telescope} \mathrm{is} \mathrm{within} \mathrm{the} \mathrm{state} \mathrm{of} \mathrm{the} \mathrm{art} \mathrm{for}$ space telescopes. 
We chose a modified Three Mirror Anastigmat (TMA) as the baseline optical design. This design allows wide-field imaging for General Astrophysics applications and a high-quality narrow field at the Cassegrain focus. The various instrument apertures are spread around the focal plane and the light is sent into a given instrument by steering the telescope in the manner of HST.

\section{KEY SCIENCE GOALS}

The science enabled by the New Worlds Observer is extensive and groundbreaking. With current and near-term technology, we can make great strides in finding and characterizing planets in the habitable zones of nearby stars. The key science goals of NWO are: 1) discover dozens of Earth-like planets in the Habitable Zones (HZ) of nearby stars with a total search completeness of 30; 2) characterize the planets we find using time-resolved photometry, spectroscopy, and polarimetry, giving us information such as atmospheric conditions, internal structure, mass estimates, and signs of life; 3) study other aspects of the extrasolar system including giant planets, planetesimal belts, and exozodiacal dust; and 4) conduct a large range of astronomical research $\sim 70 \%$ of the time, while the NWO starshade is moving from target to target.

Once exoplanets have been discovered, detailed observations such as time-resolved photometry, spectroscopy, and polarimetry will reveal the true nature of these planets and the systems in which they were born. The physical properties of exoplanets can be characterized using visible-band, reflected starlight which depends on the size of the planet, the distance between the planet and the star, the composition and structure of the planet's atmosphere and surface, the wavelength of the observation, etc.

Spectroscopy of terrestrial exoplanets will quickly reveal a wealth of information about the planet's atmospheric and surface conditions including habitability or even the presence of life. Water, carbon dioxide, oxygen, methane, ozone, and ammonia give the key signatures. Water is the necessary ingredient for the types of life found on Earth and it has played an intimate, if not fully understood, role in the origin and development of life on Earth. The presence of carbon-dioxide would indicate (1) that carbon is available for the biosphere, (2) a greenhouse effect, and (3) the possibility of climate regulation via carbon cycling between the atmosphere and hydro/geosphere. A large amount of oxygen in a terrestrial atmosphere would be extremely interesting; oxygen is so chemically reactive that it must be continuously produced at enormous rates to persist. $\mathrm{O}_{2}$ in the Earth's atmosphere is the result of continuous input from the biosphere [6].

A simulated spectrum of the Earth at 10 pc, viewed for 50 hours by NWO, is shown in Figure 5. All known sources of noise are included. Clearly visible in the spectrum is the rise to short wavelength, indicating Rayleigh scattering. Toward the red end are strong absorption features of water, indicative of oceans and clouds. Most exciting is the presence of biomarkers such as absorption lines from molecular oxygen and an absorption edge from ozone in the near ultraviolet. These features in the spectrum of the Earth arise solely as a byproduct of plant life.

An analysis of a planet's color, brightness variability, and spectrum provides an estimate of the planet's reflectivity, or albedo. From this, the planetary radius can possibly be derived as well as an estimate of its density (rocky planets tend to have much lower albedo than gas giants). This classification system provides a method to estimate planetary mass. While measuring the mass of the planet is an important parameter for detailed modeling, the most important information regarding habitability is gained through direct observation. Measurement of mass should follow planet detection and classification, as opposed to being a necessary first step.

The full suite of astrophysical techniques will be available for exoplanet observations. We can make rough measurements of atmospheric density from Raleigh scattering. Photometric monitoring could reveal surface variations for planets with relatively transparent atmospheres [7]. A high-resolution spectrometer might be used to capture a detailed spectrum of a particularly interesting planet. Similarly, 


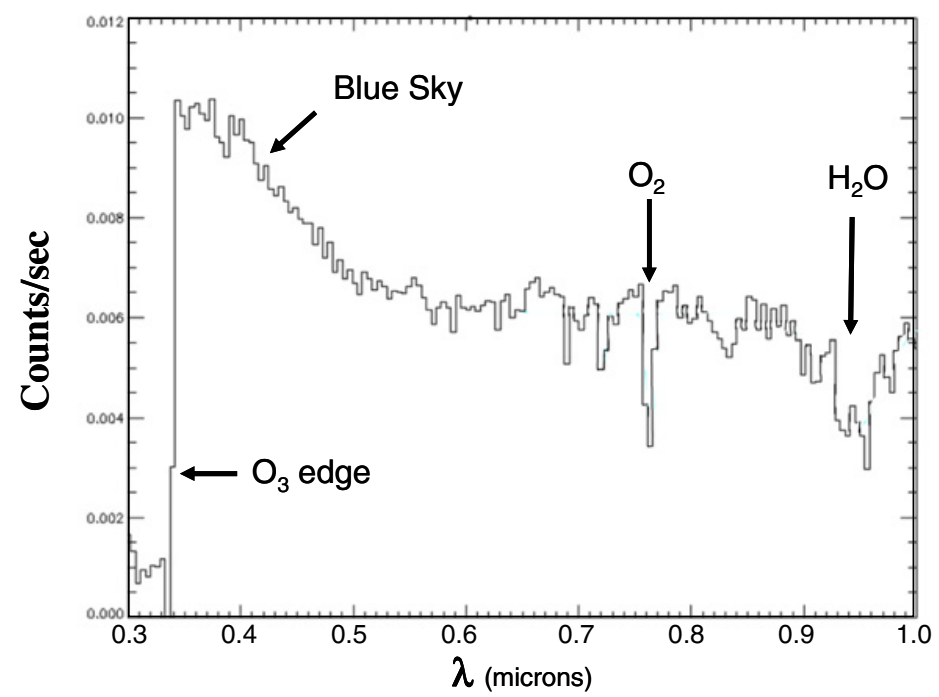

Figure 5. The spectrum of the Earth at $10 \mathrm{pc}$ as seen by NWO. Note the prominent water and oxygen absorption lines and the ozone edge in the near UV.

other general astrophysics (GA) instruments might be used to characterize planets in special circumstances.

Since NWO will have a large field of view of $\sim 0.2$ square arcminutes, we will discover outer planets and diffuse emission while searching the $\mathrm{HZ}$ of the star. The detection, characterization, and orbit determinations of gas and ice giants in the outer parts of planetary systems will provide important clues about the system's long-term dynamical evolution. NWO will provide reliable statistics on the presence of ice and gas giants in long-period orbits in mature planetary systems, estimates of disk lifetimes, etc. Given the parameters observable with NWO, it will be possible to differentiate between and constrain models of planet formation and evolution.

We must also carefully consider diffuse emission from interplanetary dust in the extrasolar systems. This exozodiacal dust (or "exozodi") is crucial, both for its science return and as a source of background noise.

The amount of exozodi is typically quantified by the fractional infrared luminosity $\left(L_{I R} / L_{*}\right)$ which is proportional to the dust mass, though other factors like grain properties affect it. Currently known exozodi disks (better known as debris disks) have fractional infrared luminosity [6] $\left(L_{I R} / L_{*}\right) \approx 10^{-3}$ $10^{-5}$. The zodiacal dust interior to our asteroid belt has $L_{I R} / L_{*} \cong 10^{-7}$, which we call 1 "zodi". We are not currently able to detect this amount of dust around other stars; this can only be done with highcontrast direct imaging. Since NWO has no outer working angle and produces zero distortions in the field, exozodiacal light and debris disks will be optimally imaged by this system.

The distribution of exozodiacal light is a sensitive tracer of the system's orbital dynamics. Planetary orbital resonances will be displayed as gaps and enhancements in the dust. Tiny planets, too small to be seen directly, should leave distinct marks. The observed dust distribution gives us critical information like the inclination of the system's ecliptic plane (Fig. 6). By eye, one can place an ellipse over the system, estimating the orientation of the plane. Then, concentric ellipses may be drawn about the central star and those that pass through a planet show the orbit of that planet under the assumption of circularity. Exozodiacal light has the potential to give us a first estimate of the orbit of each planet from a single image. Revisits will determine other planet orbit parameters. 


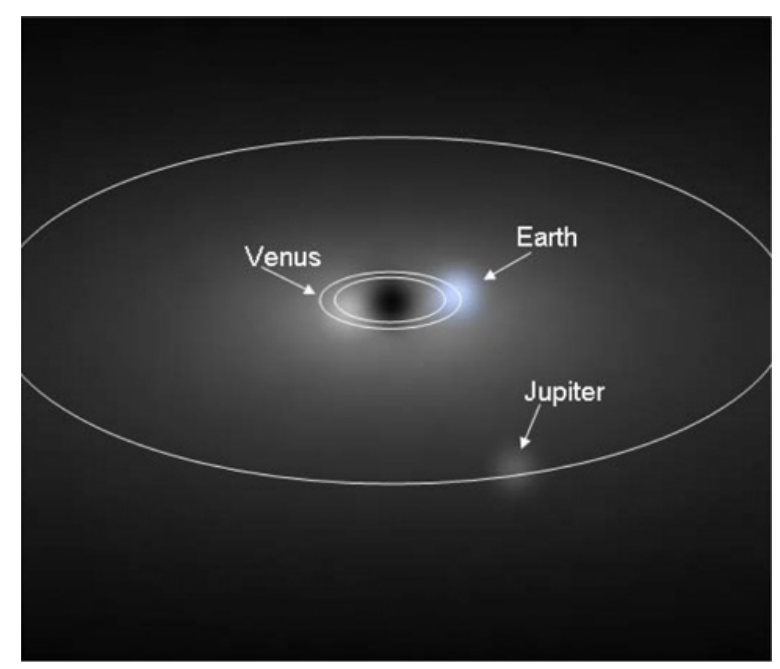

Figure 6. The exozodiacal light can help estimate the inclination of the system and therefore constrain planet orbits. This image shows a simulation of a hypothetical system with three planets - Venus, Earth and Jupiter. The exozodiacal light has total brightness equal to our own, but is more spatially extended.

Zodiacal and exozodiacal dust also create a background flux that is mixed with the planet signal in both images and spectra. Even if nearby systems have exozodi levels no greater than the Solar System level, the zodiacal and exozodiacal background will be the largest source of noise for most terrestrial planet targets, assuming the starlight is suppressed to $\sim 10^{-10}$. The surface brightness of the exozodi is the main factor controlling how long it takes to detect an exoplanet that is buried in it. We know very little about exozodi levels in nearby stellar systems. However, NWO is quite robust against the presence of many zodis of dust in the extrasolar system. A useful benchmark goal is $\mathrm{S} / \mathrm{N}=10$ on an Earth-like planet in a Solar System twin at $10 \mathrm{pc}$ viewed at a $60^{\circ}$ inclination, which NWO can achieve in 3.3 hours. Even if there is 10 zodi in this system $\left(\sim 19 \mathrm{mag} / \operatorname{arcsec}^{2}\right.$ at the planet location), NWO can image the Earth twin in less than a day.

\section{SUMMARY OF FINDINGS:}

- NWO is capable of studying thirty complete habitable zones given a contrast limit of $10^{-11}$ and inner working angle of 50mas.

- Spectra of all major planets in a Solar system twin at $10 \mathrm{pc}$ can be obtained in less than 24 hours at $\mathrm{R} \sim 100$ over a wavelength range of 0.1 to $1.1 \mu \mathrm{m}$, due to the high throughput of the NWO system.

- The wavelength range of NWO is capable of obtaining the ozone edge, water lines, and some methane lines, making the detection of life possible.

- The design of the telescope is independent of the design of the starshade. A diffraction-limited UV/O/IR telescope like HST can be used, with no additional constraints from the starshade. 70\% of the telescope time is available for general astrophysics during the time that the starshade is traveling to the next target star.

- NWO does not require invention of new technology: all the elements of the technologies used exist, including the deployment and telescope-to-starshade alignment systems, but they need to be integrated into a working system.

- Starshades provide a versatile architecture. They may be expanded, extended, and upgraded for future missions.

- A starshade can effectively be used with the upcoming James Webb Space Telescope. This represents the fastest, lowest cost route to acquiring spectra of Earth-like planets. 
EPJ Web of Conferences

\section{References}

[1] The NWO ASMCS Study Report \& Appendices, http://newworlds.colorado.edu/

[2] Spitzer, L. 1962, American Scientist, 50, 473

[3] Marchal, C. 1985, Acta Astronautica, 12, 195

[4] Copi, C.J. \& Starkman, G. 2000, ApJ, 532, 581

[5] Cash, W. 2006, Nature, 442, 51

[6] Lovelock, J. E., 1979, "A New Look at Life on Earth", Oxford University Press

[7] Oakley, P., Cash, W., \& Turnbull, M. 2008, SPIE, 7010, 70103Y 